\title{
Estudio de la difusión atómica en el intermetálico FeAl mediante Monte Carlo cinético
}

\author{
E. Manrique Castillo ${ }^{* 1}$, J. Rojas Tapia ${ }^{1}$ y E. Torres Tapia ${ }^{1}$ \\ ${ }^{1}$ Facultad de Ciencias Físicas, Universidad Nacional Mayor de San Marcos, A.P. 014-0149, Lima, Perú
}

Recibido 20 febrero 2011 - Aceptado 30 octubre 2011

\begin{abstract}
La difusión atómica en el intermetálico FeAl ordenado con estructura B2 se estudia mediante el método Monte Carlo cinético. Usamos las interacciones entre pares hasta los segundos vecinos más próximos con condiciones de frontera periódicas. Determinamos las constantes de difusión en función de la temperatura. Calculamos la función de autocorrelación que revela la vacante que efectúa saltos altamente correlaciones en la red a bajas temperaturas y los átomos que saltan a posiciones de su propia subred a temperaturas moderadas.
\end{abstract}

Palabras claves: Monte Carlo Cinético, difusión, FeAl, energía de activación..

\section{Kinetic Monte Carlo study of atomic diffusion in the FeAl intermetallic compound}

The atomic diffusion in the FeAl intermetallic compound with $\mathrm{B} 2$ ordered structure were studied by the kinetic Monte Carlo method. We use the pair interactions up to second neighbors with periodic boundary conditions to avoid surface effects. Diffusion constants was determined as a function of temperature, covering both ordered and disordered phases. In addition, we calculate the autocorrelation function which shows first, highly correlated jumps of the vacancy in the lattice at low temperatures and second, the atoms that jump to positions of its own sublattice at moderate temperatures.

Keywords: Kinetic Monte Carlo, diffusion, FeAl, activation energy.

Un sistema binario A-B se considera ordenado si la estructura atómica se describe mediante 2 o más subredes, que son ocupadas predominantemente por cierto tipo de átomos. En tales sistemas, bajo ciertas condiciones y determinadas concentraciones estequiométricas, se realiza la ordenación de largo alcance, formándose de esta manera las superestructuras. Las superaleaciones en base al intermetálico FeAl pertenece a la clase de materiales con excelentes propiedades mecánicas y alta resistencia a la corrosión y la oxidación a altas temperaturas [1-3]. La estructura ordenada es responsable de sus propiedades únicas, especialmente a elevadas temperaturas, debido a la existencia de orden de largo alcance que involucra grandes superredes, los cuales reducen el movimiento de las dislocaciones y movilidad de los átomos. El conocimiento del comportamiento de la difusión es de vital importancia para la producción y uso práctico de estos materiales estructurales, debido a que procesos tales como la transformación de fases, la deformación y el crecimiento están íntimamente rela- cionados con el transporte atómico. Los fenómenos de difusión en las aleaciones intermetálicas ordenadas B2 todavía no está comprendido totalmente [4-6]. Es aceptado que la difusión procede vía la migración de defectos vacantes. Los principales defectos puntuales en las aleaciones ordenadas son las vacantes térmicas y los defectos antisitios (DAS) de tipo A y $\mathrm{B}^{1}$. Además, un rol muy importante juegan los defectos triples que constan de 2 A-vacantes y un A-DAS [5,7,8]. La información experimental sobre el rol de las vacantes y los mecanismos de difusión se obtienen usando espectroscopia cuasielástica Mössbauer de ensanchamiento de línea [9], que nos proporciona los vectores de saltos individuales para un modelamiento apropiado [10,11]. Los experimentos de la difusión atómica en la fase B2, en particular en $\mathrm{FeAl}$ ordenado, permite observar que la difusión ocurre vía saltos a los primeros vecinos más próximos (pvm) y que durante la migración de las vacantes no se altera el orden de largo alcance. Esto ha motivado que algunos autores sugieran que la difusión atómica resulta de las

\footnotetext{
*emanriquec@unmsm.edu.pe

${ }^{1}$ El defecto antisitio A ocurre cuando un átomo A ocupa el sitio de B en la subred $\beta$.
} 
secuencias de los saltos altamente correlacionadas de la vacante.

Dos mecanismos se han propuesto para vencer las barreras energéticas, que operan en contra de la difusión, sin alterar el orden de largo alcance en las aleaciones ordenadas de tipo B2. El primer mecanismo involucra saltos a los siguientes vecinos más próximos (svp). Donaldson y Rawlings [12], basados en un estudio de difusión de trazas, sugieren un mecanismo svp para los átomos de $\mathrm{Ni}$ en el compuesto $\mathrm{B} 2$, NiGa. El segundo mecanismo se refiere a movimientos cíclicos donde los átomos efectúan una serie de saltos a los vecinos más próximos siguiendo un camino definido. El ciclo de los seis saltos, postulado por Elcock y McCombie [13], ver figura 1, es el más conocido.

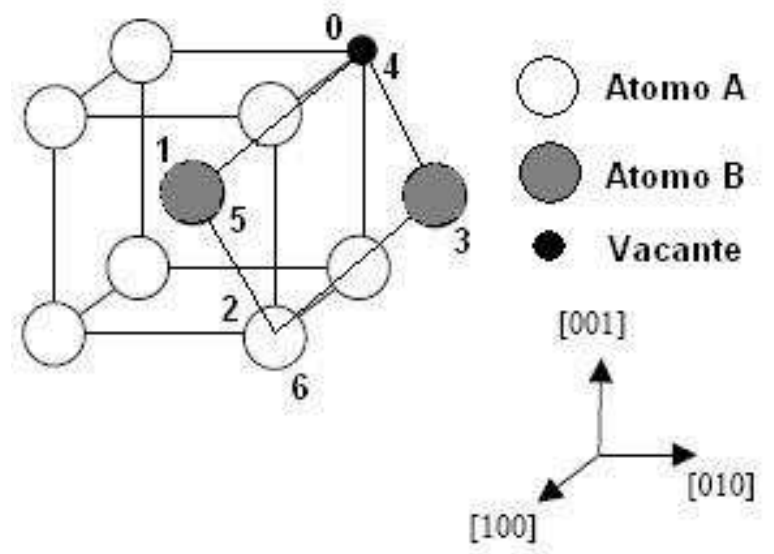

Figura 1: Ciclo de los seis saltos de la vacante a un tercer vecino más próximo [13].

En el presente trabajo estudiamos el proceso de difusión en el compuesto intermetálico $\mathrm{FeAl}$ en un amplio rango de temperaturas, que incluye las fases ordenadas B2 y desordenada A2, con la finalidad de determinar los coeficientes de difusión y otros parámetros termodinámicos. El método que usamos es el Monte Carlo Cinético conjuntamente con el algoritmo de tiempo de residencia, donde la migración átomica resulta de los intercambios con una simple vacante con uno de sus vecinos más próximos en cada paso de Monte Carlo (MC).

\section{El Modelo atomístico cinético}

El modelo B2 del intermetálico FeAl está conformado por dos subredes cúbicas simples superpuestas: la primera subred $\alpha$ está ocupada por los átomos $\mathrm{A}(\mathrm{Fe})$, mientras que, la segunda subred $\beta$ está ocupada por los átomos $\mathrm{B}(\mathrm{Al})$. Usamos dos sistemas de coordenadas cartesianos para determinar la posición de los nodos de la red cúbica simple. Cada uno de los sistemas de coordenadas establece la posicion de los nodos de cada subred; estos sistemas coordenados estan separados por el vector $a(0.5,0.5,0.5)$, donde $a$ es la dimensión de la celda unitaria de la red. La red cúbica simple consta de $N=2(L \times L \times L)$ sitios, donde $L$ es el número de celdas en cada dimensión. La aleación binaria consiste de $N_{\mathrm{A}}$ átomos $\mathrm{A}, N_{\mathrm{B}}$ átomos $\mathrm{B}$ y una vacante, tal que $N=N_{\mathrm{A}}+N_{\mathrm{B}}+1$ y $N_{\mathrm{B}}=N_{\mathrm{A}}-1$.

Usamos el modelo de Kim [14], para definir las energías como energías de interacción por par $\epsilon_{X Y}^{i}$ entre átomos ubicados en los sitios vecinos más próximos, donde $\mathrm{X}, \mathrm{Y}$ son iguales a $\mathrm{A}$ o $\mathrm{B}$, e $i=1,2$ indican los primeros y segundos vecinos más próximos, respectivamente.

La difusión de los átomos $\mathrm{A}$ y $\mathrm{B}$ ocurren por medio de intercambios, activados térmicamente, de la posición de los átomos (A o B) con la vacante (V). De acuerdo a la teoría de la razón o tasa de intercambio [15], la frecuencia de intercambio del átomo $\mathrm{X}$ con la vacante $\mathrm{V}$ (donde $\mathrm{X}=\mathrm{A}$ o $\mathrm{B}$ ) está dado por

$$
\Gamma=\nu_{0} e \exp \left[-\frac{\Delta E_{\mathrm{XV}}}{k T}\right]
$$

donde $\nu_{0}$ es una frecuencia de prueba de salto, $\Delta E_{X V}$ es la barrera de activación, es decir, la energía necesaria para quebrar el par $\mathrm{X}-\mathrm{V}$ de su entorno y llevar al átomo $\mathrm{X}$ a una posición punto silla, donde su energía de interacción con el sistema es $E_{s}$.

Asumiendo que la barrera o energía de activación depende solo de las especies químicas que se encuentran en las cercanías de la vacante, su valor es calculada usando el modelo del enlace quebrado [16],

$$
\Delta E_{\mathrm{XV}}=E_{s}-\sum_{i=1}^{2} \sum_{\substack{p \in \\ n_{i}(\mathrm{X})}} \epsilon_{\mathrm{X}_{p}}^{i} .
$$

La suma en la ecuación (2) se extiende sobre todos los átomos en la $i$-ésima esfera de coordinación al rededor del átomo X. Por simplicidad, $\nu_{0}$ y $E_{s}$ son constantes, puesto que a una temperatura dada el valor de $E_{s}$ solo contribuye definiendo la escala de tiempo absoluto.

Por otro lado, cuando $E_{s}$ se expresa en función de las energías de interacción $\epsilon_{\mathrm{XY}}^{i}$ y los números de coordinación $z_{i}$ de la $i$-ésima esfera de coordinación, mediante

$$
E_{s}=\left[\left(z_{1}-1\right)\left(\epsilon_{A A}^{1}+\epsilon_{B B}^{1}\right)+z_{2}\left(\epsilon_{A A}^{2}+\epsilon_{B B}^{2}\right)\right] / 2,
$$

tenemos que las energías de ordenación están dadas por

$$
\epsilon_{i}=\epsilon_{A A}^{i}+\epsilon_{B B}^{i}-2 \epsilon_{A B}^{i}
$$

y las energías asimétricas por

$$
u_{i}=\epsilon_{A A}^{i}-\epsilon_{B B}^{i},
$$

entonces la energía de activación de la ecuación (2) puede ser reescrita como

$$
\Delta E_{\mathrm{XV}}^{j}=\frac{1}{2} \sum_{i=1,2} \epsilon_{i} n_{\mathrm{Y}}^{i}+u_{i} \theta_{\mathrm{X}} n_{\mathrm{X}}^{i}
$$


donde $(\mathrm{X}, \mathrm{Y})=(\mathrm{A}, \mathrm{B})$ o $(\mathrm{B}, \mathrm{A}), \theta_{\mathrm{B}}=-1, \theta_{\mathrm{A}}=1$ y $n_{\mathrm{X}}^{i}, n_{\mathrm{Y}}^{i}$ son los números de átomos de tipo $\mathrm{X}$ e $\mathrm{Y}$ en la $i$-ésima esfera de coordinación alrededor del sitio ocupado por el átomo X, que será intercambiado con la vacante.

En nuestro caso, consideramos las interacciones hasta los segundos vecinos más próximos, el modelo presenta una transición de la fase desordenada A2 a la fase ordenada B2. Para los propósitos del presente trabajo, los parámetros empleados son $\left(\epsilon_{1}, \epsilon_{2}, u_{1}, u_{2}\right)=$ $(0.03,-0.04,0.04,0) \mathrm{eV}$, como continuación de las simulaciones previas de la aleación FeAl [17].

\section{Algoritmo del tiempo de residencia}

En este trabajo estudiamos las propiedades cinéticas a bajas temperaturas usando el algoritmo del tiempo de residencia [18]. Existe una derivación probabilística de este algoritmo propuesta por Novotny [19], donde se demuestra la equivalencia del algoritmo del tiempo de residencia con el algoritmo de Metropolis.

Exponemos brevemente, cómo se construye el camino seguido por una vacante mediante este algoritmo. Para salir de una cierta configuración microscópica $\mathcal{C}$, el sistema puede sufrir $z_{1}$ transiciones correspondientes a los $z_{1}$ posibles saltos de la vacante a los vecinos más próximos con frecuencias $\Gamma_{1}(\mathcal{C}), \Gamma_{2}(\mathcal{C}), \ldots, \Gamma_{z_{1}}(\mathcal{C})$.

Después de un tiempo $t$, la probabilidad de que el sistema se mantenga aún en la configuración $\mathcal{C}$ está dado por

$$
p(\mathcal{C}, t)=\exp \left[-\frac{t}{\tau(\mathcal{C})}\right]
$$

donde $\tau(\mathcal{C})$ es el tiempo de residencia promedio en la configuración $\mathcal{C}$ y está definido como

$$
\tau(\mathcal{C})=\left[\sum_{i=1}^{z_{1}} \Gamma_{i}(\mathcal{C})\right]^{-1}
$$

En cada paso de MC, un número aleatorio $r$ es generado entre 0 y $1 / \tau(\mathcal{C})$, mientras que, la transición $j$ realizada es la que satisface la desigualdad

$$
\sum_{i=1}^{j-1} \Gamma_{i}(\mathcal{C}) \leq r<\sum_{i=1}^{j} \Gamma_{i}(\mathcal{C})
$$

y el tiempo total se incrementa en $\tau(\mathcal{C})$.

En el marco de este algoritmo, en cada intento de salto, la vacante pasa necesariamente a uno de sus $z_{1}$ primeros vecinos más próximos y el paso de MC se define igual a un salto de la vacante. Esta unidad de tiempo se denomina dimensional timeless unit, dtu y el tiempo real será proporcional al tiempo medido en dtus.

\section{Resultados y discusión}

A continuación mostramos los resultados de las simulaciones con el método Monte Carlo Cinético (MCC) del fenómeno de difusión atómica en una aleación de fase ordenada B2, considerando la fase intermetálica ordenada $\mathrm{Fe}_{50} \mathrm{Al}_{50}$. La dinámica se introdujo a través del movimiento de una sola vacante [20], con saltos permitidos a una de las ocho posiciones vecinas más próximas. El grado de orden del sistema se determina por medio del parámetro de orden de largo alcance.

El parámetro de orden de largo alcance $\eta$ está definido como

$$
\eta=\frac{1}{N}\left(\sum_{\alpha} S_{i}\left(\boldsymbol{r}_{i}\right)-\sum_{\beta} S_{j}\left(\boldsymbol{r}_{j}\right)\right)
$$

donde $S_{i}$ es la variable de espín en la posición $\boldsymbol{r}_{i}$ en la subred $\alpha$, mientras que $S_{j}$ en la posición $\boldsymbol{r}_{j}$ en la subred $\beta$.

El proceso de termalización de dos sistemas hacia el equilibrio ocurre cuando el primero parte de un estado ordenado y el segundo de un estado desordenado. Cuando ambos sistemas alcanzan el mismo parámetro de orden de largo alcance, se considera que ambos sistemas alcanzaron el estado de equilibrio a la temperatura dada.

\section{Constantes de difusión y parámetros termo- dinámicos}

La manera formal de determinar la constante de difusión de trazas por medio de simulación computacional es calculando los desplazamientos cuadráticos medios (DCM) de los átomos trazadores [21]. La constante de difusión de la vacante es calculada como [22]

$$
D_{V}=\lim _{t \rightarrow \infty}\left(\frac{1}{6} \frac{\partial}{\partial t} R_{V}^{2}\right)
$$

donde $R_{V}^{2}$ es el DCM total de la vacante después del tiempo $t$.

Tanto los átomos del tipo A como los del tipo B pueden ser considerados como trazadores, luego las constantes de difusión de las trazas $D_{\mathrm{A}}\left(D_{\mathrm{B}}\right)$ se calculan de la misma forma mediante la relación,

$$
D_{\mathrm{A}}=\lim _{t \rightarrow \infty}\left[\frac{1}{6 N_{A}} \frac{\partial}{\partial t}\left(\sum_{\mathrm{A}} R_{\mathrm{V}}^{2}\right)\right]
$$

Los valores medidos de los DCM fueron promediados sobre 60 corridas independientes. A partir de estos DCMs extraemos los valores de los coeficientes de difusión de los átomos en la aleación ordenada. En las figuras 2 y 3 se presentan los valores obtenidos de los 
DCM, correspondientes a los valores de la temperatura que incluye la región ordenada y desordenada. La temperatura crítica para el sistema estudiado es igual a $T_{c}=1175 \mathrm{~K}$.
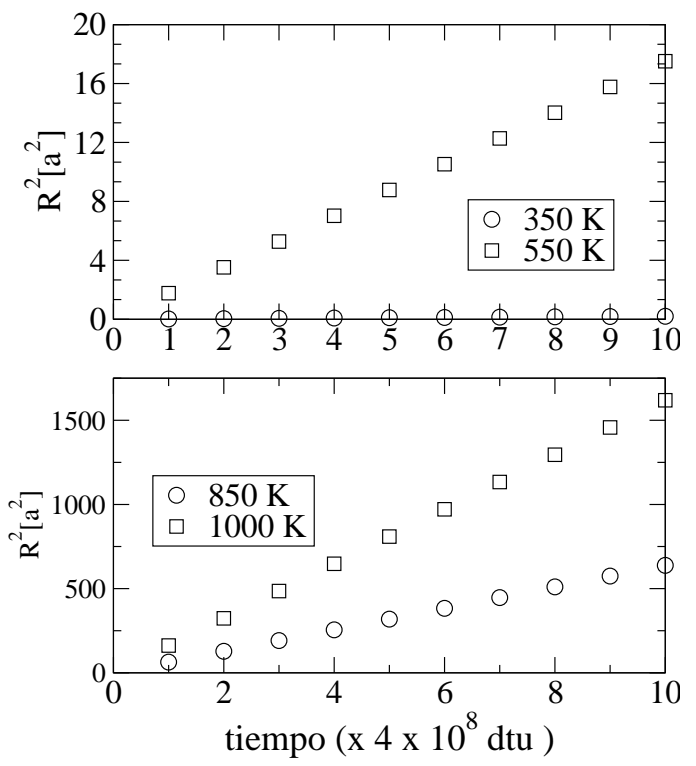

Figura 2: Variación con el tiempo del DCM en FeAl modelo con $\mathrm{L}=100$, correspondientes a temperaturas menores que la temperatura crítica $T_{c}=1175 \mathrm{~K}$.
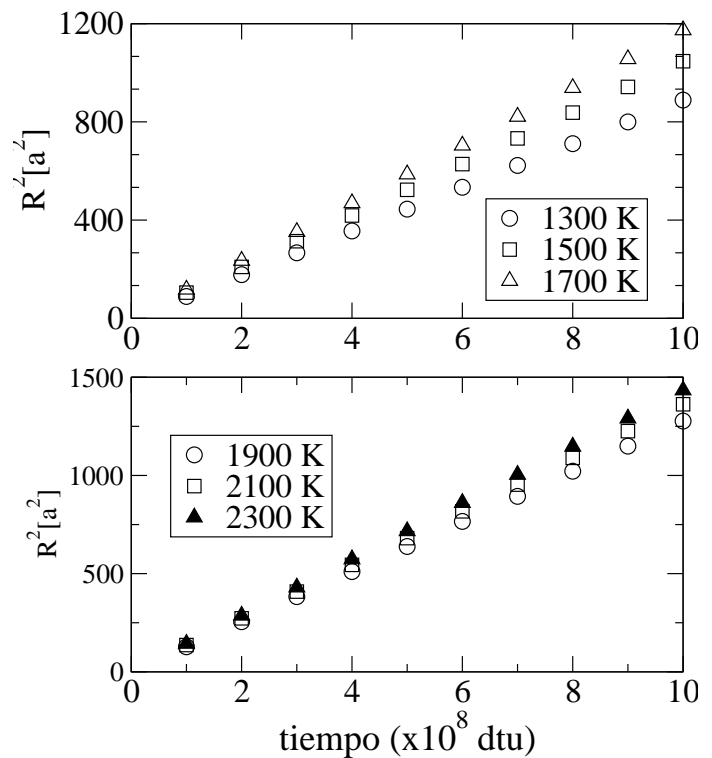

Figura 3: Variación con el tiempo del DCM en FeAl modelo con $\mathrm{L}=100$, correspondientes a temperaturas mayores que la temperatura crítica $T_{c}=1175 \mathrm{~K}$.

El coeficiente de difusión o difusividad $D_{A}$ está dado, para redes cúbicas, por $1 / 6$ de la pendiente de las rectas del DCM, similares a las figuras 2 y 3 . La pendiente de las rectas del DCM respecto a $t$ son determinados mediante el ajuste de los mínimos cuadrados.

La variación con la temperatura de los coeficientes de difusión atómica satisfacen la ecuación de Arrhenius

$$
D(T)=D_{0} \exp \left[-\frac{E_{\mathrm{A}}}{k T}\right],
$$

donde $D_{0}$ es una constante que no depende de la temperatura, $E_{\mathrm{A}}$ es la energía de activación de la difusión o energía de migración de la vacante.

La ecuación de Arrhenius, Ec.(13) en forma logarítmica representa una dependencia lineal del logaritmo del coeficiente $D(T)$ como función de $1 / T$,

$$
\ln D=\ln D_{0}-\left(\frac{E_{\mathrm{A}}}{k}\right) \frac{1}{T} .
$$

Como se puede apreciar en la figura 4, las gráficas de Arrhenius cambian de pendiente en la temperatura crítica $T_{c}$. Por lo tanto, los valores para $\ln D_{\mathrm{A}}$ pueden ser ajustados por dos lineas rectas como una función de $1 / T$ para temperaturas sobre y debajo de la $T_{c}$, permitiendo, de esta manera, determinar las energías de migración $E_{A 2}^{m}$ y $E_{B 2}^{m}$ correspondientes a las fases desordenadas y ordenadas, respectivamente.

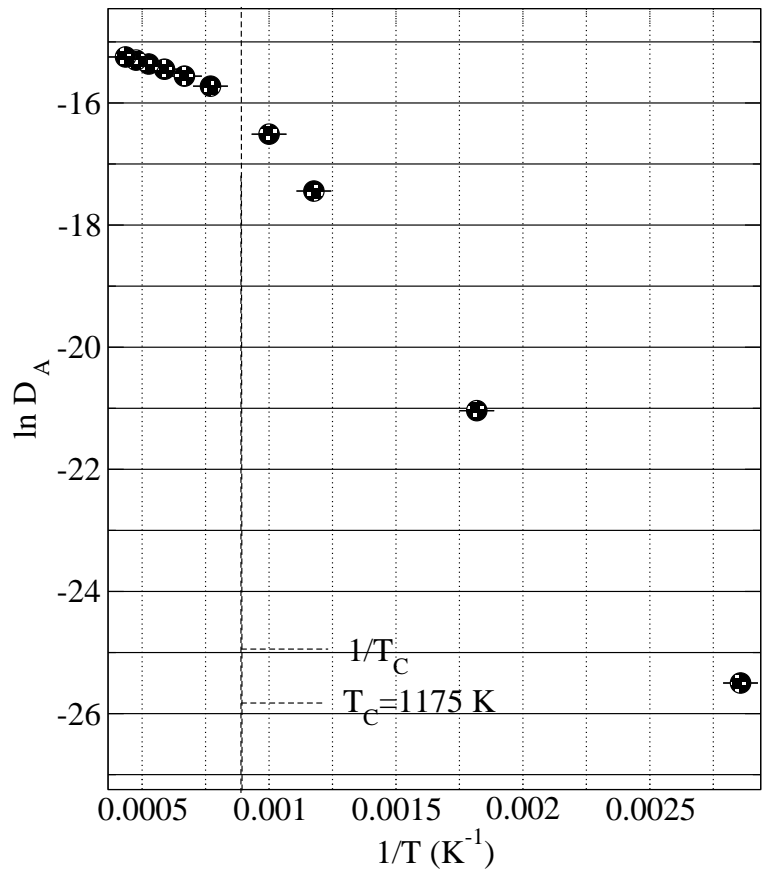

Figura 4: Gráficas de Arrhenius del logaritmo de la difusividad versus la temperatura inversa en el compuesto FeAl.

La energía de migración $E_{B 2}^{m}$ es mayor en la fase ordenada (debajo $T_{C}, E_{B 2}^{m}=0.42 \mathrm{eV}$ ) que en la fase desordenada (sobre $T_{C}, E_{A 2}^{m}=0.12 \mathrm{eV}$ ). El hecho de 
que la energía de migración $E_{A 2}^{m}$ es menor en la fase desordenada es razonable por que no hay un cambio muy significativo en el entorno de la vacante antes y después del salto.

\section{Función de autocorrelación}

A diferencia de la subsección anterior, en esta parte obtenemos evidencia directa de los saltos elementales de los átomos, relacionados con la difusión, calculando la función de autocorrelación. Los experimentos de espectroscopia Mössbauer cuasielástica [23] y la dispersión cuasielástica de neutrones [24] miden la función de autocorrelación (o la transformada de Fourier) de un átomo marcado en la aleación. La función de autocorrelación proporciona la información del átomo marcado después de un tiempo $t$, considerando que el átomo está, inicialmente, en el origen para $t=0$.

Tabla 1: Distribución de probabilidades de los átomos $\mathrm{A}$ de encontrarse en las diferentes esferas de coordinación en el intermetálico $\mathrm{FeAl}$ a la temperatura de $350 \mathrm{~K}$ respecto a diferentes instantes de tiempo, donde $\tau_{0}=5 \times 10^{8} d t u$ s.

\begin{tabular}{ccccccc}
\hline$z_{i}$ & $\tau_{0}$ & $2 \tau_{0}$ & $3 \tau_{0}$ & $4 \tau_{0}$ & $5 \tau_{0}$ & $6 \tau_{0}$ \\
\hline 0 & 0.9837 & 0.9678 & 0.9522 & 0.9370 & 0.9223 & 0.9078 \\
1 & 0.0009 & 0.0012 & 0.0019 & 0.0024 & 0.0028 & 0.0031 \\
2 & 0.0092 & 0.0182 & 0.0269 & 0.0353 & 0.0435 & 0.0515 \\
3 & 0.0049 & 0.0095 & 0.0142 & 0.0187 & 0.0231 & 0.0273 \\
4 & 0.0000 & 0.0000 & 0.0000 & 0.0007 & 0.0008 & 0.0010 \\
5 & 0.0007 & 0.0010 & 0.0018 & 0.0022 & 0.0029 & 0.0035 \\
6 & 0.0000 & 0.0000 & 0.0009 & 0.0010 & 0.0010 & 0.0011 \\
7 & 0.0000 & 0.0000 & 0.0000 & 0.0000 & 0.0000 & 0.0000 \\
8 & 0.0000 & 0.0100 & 0.0100 & 0.0014 & 0.0019 & 0.0022 \\
9 & 0.0000 & 0.0000 & 0.0002 & 0.0009 & 0.0010 & 0.0010 \\
10 & 0.0000 & 0.0000 & 0.0001 & 0.0004 & 0.0009 & 0.0010 \\
\hline
\end{tabular}

En la tabla 1 se muestran los valores de la probabilidad de encontrar un átomo $\mathrm{A}$ en la $k$-ésima esfera de coordinación en el tiempo $t$ a una temperatura de 350 K. Se observa que después de un tiempo de $30 \times 10^{8}$ dtus un $90 \%$ de los átomos A no se movieron, y si lo hicieron, regresaron a su punto inicial de partida (el origen de coordenadas propio). Se observa que la primera esfera de coordinación está vacía, mientras que el $10 \%$ restante de átomos $\mathrm{A}$ se hospedan en la $2^{\text {da }}$ y $3^{\text {era }}$ esferas de coordinación. Este resultado es importante porque confirma el mecanismo del ciclo de seis saltos (C6S) en el movimiento de los átomos [13].

En efecto, después que este ciclo se ha completado, el cristal es nuevamente ordenado y la vacante ha migrado a un segundo o un tercer vecino más próximo. Finalmente, podemos agregar que de acuerdo a los valores de la tabla, hay una preferencia de los saltos a los sitios que se encuentran como segundos vecinos más próximos sobre los saltos a los sitios que se encuentran como los terceros vecinos más próximos con una razon aproximada de $2: 1$, razón que se mantiene constante después de un tiempo $t=15 \times 10^{8} \mathrm{dtus}$.

En las figuras 5 y 6 , se presentan los histogramas con los resultados de la función de autocorrelación de los átomos de tipo A a la temperatura de $550 \mathrm{~K}$. De las figuras observamos que cuando ha transcurrido un tiempo $t=4 \times 10^{8} d t u$ s, un $35 \%$ de los átomos A no se han movido o saltaron hacia atrás a su punto de partida y solo un $3 \%$ de los átomos A alcanza la $10^{\text {ma }}$ esfera de coordinación o las mayores esferas. También es notorio que la probabilidad de encontrar el átomo A decrece con el índice de la esfera de coordinación.

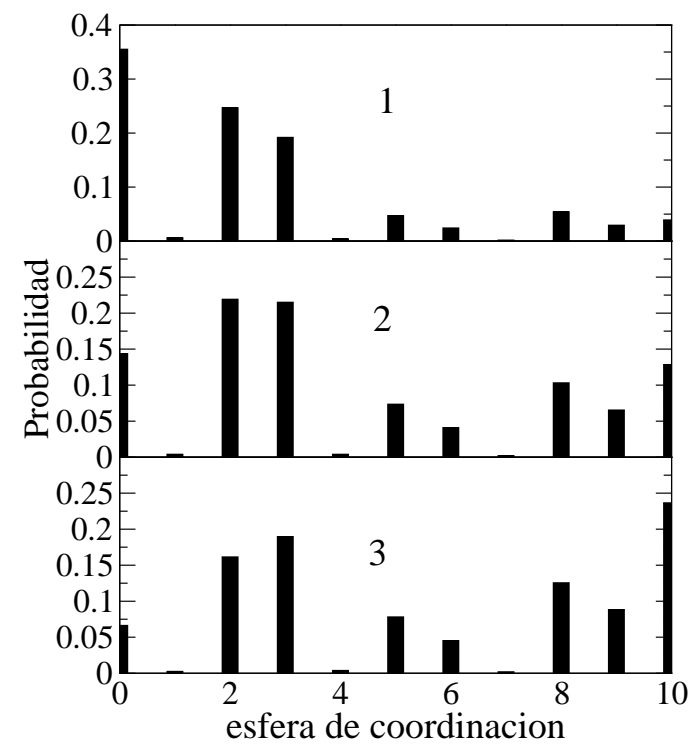

Figura 5: Probabilidades de encontrar los átomos A en las diferentes esferas de coordinación en el intermetálico FeAl ordenado a la temperatura de $550 \mathrm{~K}$. Los números 1,2 y 3 indican los instantes de tiempo $\tau, 2 \tau$ y $3 \tau$, respectivamente, donde $\tau=4 \times 10^{8} d t u$ s.

Además, resulta que la primera esfera de coordinación no está ocupada en todo instante de tiempo, por lo menos durante el intervalo que transcurre desde $t=4 \times 10^{8} d t u$ s hasta $t=24 \times 10^{8} d t u$ s; por el contrario 
durante todo ese intervalo de tiempo la segunda y tercera esferas de coordinación son ocupadas por átomos A.

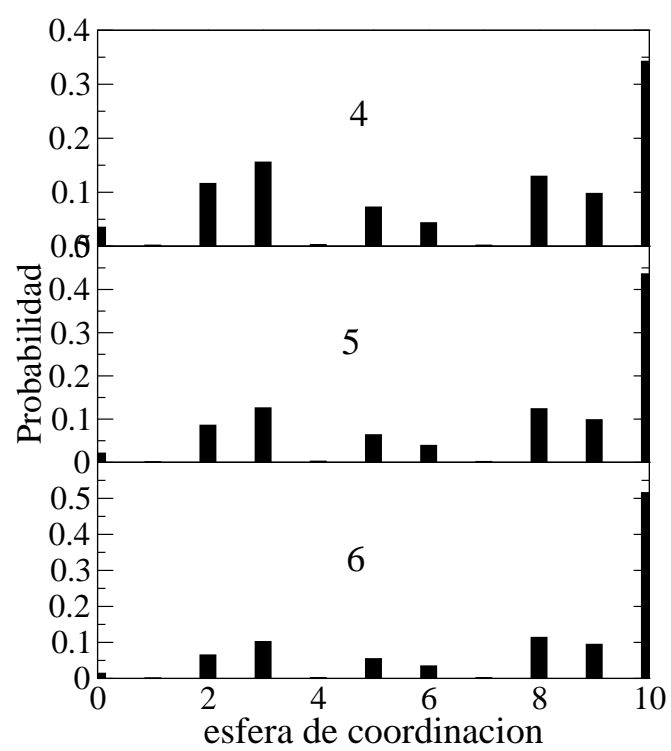

Figura 6: Probabilidades de encontrar los átomos A en las diferentes esferas de coordinación en el $\mathrm{FeAl}$ ordenado a temperatura de $550 \mathrm{~K}$. Los números 4,5 y 6 indican los instantes de tiempo $4 \tau, 5 \tau$ y $6 \tau$, respectivamente, donde $\tau=4 \times 10^{8}$ dtus.

La característica más resaltante es que después del tiempo $t=24 \times 10^{8} d t u$ s, la ocupación de la tercera esfera de coordinación excede claramente a la ocupación de la segunda esfera por los átomos. Por otro lado, hay una preferencia de saltos de los átomos de Fe entre sitios de su propio subred. Esta preferencia de saltos a los sitios que se encuentran como terceros vecinos más próximos (saltos [110]) sobre los saltos a los sitios que se encuentran como segundos vecinos más próximos (saltos [100]) ocurre con una razón de probabilidad $W_{[110]} / W_{[100]}=1.6$.

Como la primera esfera de coordinación está desocupada en todo instante y observando que la segunda y la tercera esfera de coordinación están siempre ocupadas, entonces caben dos posibilidades en la dinámica de la vacante. La primera posibilidad es que la vacante realiza el ciclo de los seis saltos, mientras que en la segunda, la vacante salta a segundos o terceros vecinos más próximos.

Los experimentos de espectroscopia Mössbauer cuasielástica para el caso de $\mathrm{B} 2 \mathrm{FeAl}$, mostraron que los átomos de Fe saltan efectivamente entre nodos de su propia subred. Dichos saltos son mayormente a los terceros vecinos más próximos y, parcialmente, a los segundos vecinos más próximos.

Además, dichos experimentos proporcionan la evi- dencia, para temperaturas intermedias, de que los saltos son combinaciones de dos saltos a primeros vecinos más próximos. Entonces, por lo anteriormente mencionado, afirmamos que los átomos $\mathrm{A}, \mathrm{Fe}$, saltan a los sitios de su propia subred, es decir, a la $2^{\text {da }}$ y $3^{\text {era }}$ cáscara de vecinos más próximos, por medio de la combinación de dos saltos a los primeros vecinos más próximos. Resultando un tiempo corto de ocupación de un sitio antiestructura en la subred del Aluminio. Por último, y por eso no menos importante, podemos decir que la preferencia de los saltos [110] sobre los saltos [100] está reflejado en el hecho de que existe, aproximadamente independiente del tiempo, mayor ocupación de la tercera esfera de vecinos más proximos con respecto de la segunda esfera de vecinos más próximos, en la razón promedio de 1.6, como se puede observar en la figura 7 .

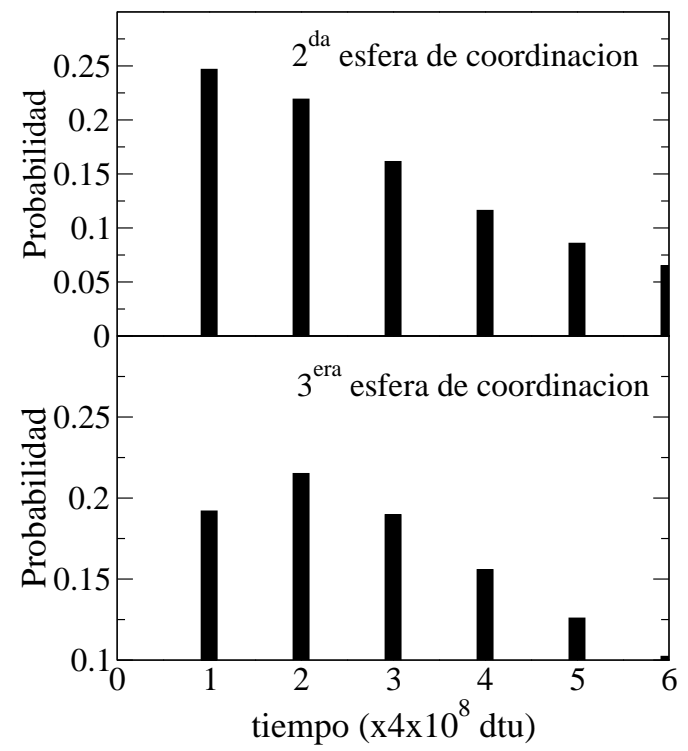

Figura 7: Variación temporal de las probabilidades de encontrar un átomo A en la $2^{\text {da }}$ y $3^{\text {ra }}$ esferas de coordinación en la fase ordenada del intermetálico FeAl a la temperatura de $550 \mathrm{~K}$.

\section{Conclusiones}

Mediante la simulación con el método Monte Carlo cinético estudiamos los procesos y mecanismos de difusión de los componentes en el intermetálico FeAl. El cálculo del desplazamiento cuadrático medio de los átomos a diferentes temperaturas permitió determinar los coeficientes de difusión y la energia de activación de la migración de los átomos $\mathrm{A}, \mathrm{Fe}$ en la aleación modelo $\mathrm{FeAl}$. La energía de migración del Fe en la fase ordenada $\mathrm{B} 2$ es $0.42 \mathrm{eV}$, mientras que en la fase desordenada A2 es $0.12 \mathrm{eV}$. 
Se encontró que debajo de la temperatura crítica la vacante efectúa movimientos altamente correlacionados, lo cual está en buena conformidad con los resultados experimentales. Finalmente, el análsis de la función de autocorrelación de los átomos A permitió mostrar que a bajas temperaturas el principal mecanismo de difusión es el ciclo de los 6 saltos. A temperaturas mo- deradas el mecanismo de difusión de los átomos A es el salto entre posiciones dentro de la propia subred, a la segunda o tercera esfera de coordinación; pero estos saltos no son directos, sino por medio de la combinación de dos saltos consecutivos a primeros vecinos más próximos.

\section{Referencias}

[1] J. Caulfield y J. M. Tien; Superalloys, Supercomposites and superceramics., Academic Press Inc., Boston (1989).

[2] O. Semenova, R. Krachler y H. Ipser; Solid State Sciences 10, 1236 (2008).

[3] Y. Cui, R. Kato, T.Omori, I. Ohnuma, K. Oikawa, R. Kainuma y K. Ishida; Scripta Materialia 62, 171 (2010).

[4] G. Bester, B.Meyer, M. Fahnle y C.L. Fu; Materials Science and Engineering A 323, 487 (2002).

[5] T. Haraguchi y M. Kogachi; Materials Science and Engineering A 329, 402 (2002).

[6] R. Nakamura, K. Takasawa, Y.Yamazaki y Y. Iijima; Intermetallics 10, 195 (2002).

[7] J. L. Jordan y S. C. Deevi; Intermetallics 11, 507 (2003).

[8] J. H. Schneibel y L. M. Pike; Intermetallics 12, 85 (2004).

[9] A. Hanc, J. Kansy, G. Dercz y I. Jendrzejewska; J. of Alloys and Compounds 48, 84 (2009).

[10] B. Sepiol R. Felwish y G. Vogl; Acta Metall. 43 , 2033 (1994).

[11] B. Sepiol; Defect Diffusion Forum 1, 125 (1995).
[12] R. D. Rawlingsy A. T. Donaldson; Acta Metall. 24, 285 (1976).

[13] C. W. McCombie y E. W. Elcock; Phys. Rev. B. 109, 605 (1958).

[14] S. M. Kim; J. Phys. Chem. Solids 49, 65 (1988).

[15] C. P. Flynn; Point and Defects Diffusion, Clarendon, Oxford (1972).

[16] G. Martin; Phys. Rev. B 41, 2279 (1990).

[17] G. Martin, F. Haider, M. Athenes y P. Bellon; Acta Mater. 44, 12 (1996).

[18] E. Elcock y W. Young; Proc. Phys. Soc. 89, 735 (1966)

[19] M. A. Novotny; Computer in Physics 9, 1 ( 1995).

[20] C. Frontera, E. Vives y A. Planes; Z. Phys. B 96, 79 (1994).

[21] L.P. Van der Meij, H. Bakker y N. Stolwijk; Nucl. Metall., Metall. Soc. AIME (US) 20, 96 (1976).

[22] D.J. Srolovitz, L. Zhao y R. Najafabadi; Acta Mater. 44, 2737 (1996).

[23] Gero Vogl; Mossbauer Spectroscopy Applied to Magnetism and Materials Science, Vol. 2, Plenum Press, New York (1996).

[24] M. Bée; Quasielastic Neutron Scattering, Adam Gilger, Bristol (1988). 